\title{
Synthesis of 3-alkyl-6-methyl-1,2,4,5-tetrazines via a Sonogashira-type cross-coupling reaction
}

Enric Ros, ${ }^{a}$ Amparo Prades, ${ }^{a}$ Dominique Forson, ${ }^{a}$ Jacqueline Smyth, ${ }^{a}$ Xavier Verdaguer, ${ }^{a, b}$ Lluís Ribas de Pouplana*a,c and Antoni Riera*a,b

a. Institute for Research in Biomedicine (IRB Barcelona), Barcelona Institute of Science and Technology, Baldiri Reixac 10, 08028 Barcelona, Spain.

b. Departament de Química Inorgànica i Orgànica, Secció Orgànica. Universitat de Barcelona, Martí i Franquès 1, Barcelona E-08028, Spain.

c. Institució Catalana de Recerca i Estudis Avançats (ICREA), Passeig Lluís Companys, 23, Barcelona 08010, Spain.

e-mail: antoni.riera@irbbarcelona.org; lluis.ribas@irbbarcelona.org.

1,2,4,5-Tetrazines have become extremely useful tools in chemical biology. However, the synthesis of some challenging substrates such as 3,6-dialkyl-1,2,4,5-tetrazines is still a limitation for the widespread use of this class of compounds. Herein we describe an efficient route to these compounds based on the Sonogashira coupling of 3-bromo-6-methyl-1,2,4,5-tetrazine with terminal alkynes. The preparation of the starting reagent has been also optimized. The alkynyl products have been used as intermediates for the synthesis of dialkyl-tetrazines through a sequence of hydrogenation and re-oxidation with unprecedented yields. The synthetic applicability of this new approach is showcased through the preparation of several unnatural amino acids bearing alkynyl- and alkyl-1,2,4,5-tetrazine fragments.

\section{Introduction}

Throughout the $20^{\text {th }}$ Century, the interest for 1,2,4,5-tetrazines remained almost completely limited to natural product synthesis, ${ }^{1,2}$ coordination chemistry ${ }^{3}$ and explosive applications. ${ }^{4,5}$ However, with the advent of click chemistry in the beginning of the $2000 \mathrm{~s}^{6,7}$ and their first use as bioorthogonal reagents with strained alkenes through the inverse electron-demand Diels-Alder (iEDDA) cycloaddition in $2008,8,9$ the interest in tetrazines increased exponentially. Their use as advantageous research tools in live cell imaging, ${ }^{10}$ bioconjugation experiments ${ }^{11,12}$ or modern drug discovery techniques ${ }^{13}$ has exemplified their huge potential. Furthermore, therapeutic applications for nuclear medicine, ${ }^{14}$ in vivo PROTAC assembly ${ }^{15}$ or prodrug activation ${ }^{16,17}$ have expanded their use in the pharmaceutical industry. The iEDDA cycloaddition endows some characteristics that allow its widespread application as a unique chemical biology tool: high selectivity, superfast kinetics in water and absence of metal catalysis. ${ }^{18}$

Despite great efforts directed towards the development of robust synthetic methods for the generation of tetrazines, the preparation of certain compounds is still challenging. For instance, tetrazines are typically synthesized through the condensation of two nitriles in the presence of hydrazine hydrate, followed by an oxidation step of the obtained dihydrotetrazine. ${ }^{18,19}$ While this is a useful strategy for aromatic nitriles, it is quite inefficient in the case of aliphatic, inactivated nitriles to form the corresponding (alkyl)tetrazines. ${ }^{19,20}$ Although the addition of $\mathrm{Ni}^{\prime \prime}$ or $\mathrm{Zn}$ "salts, was a great improvement, ${ }^{21}$ the synthesis of asymmetrically substituted tetrazines remains a challenge due to the generation of a statistical mixture of products that require difficult purification. Furthermore, the use of anhydrous hydrazine to improve the yields is problematic and the required high temperatures limit the presence of some functional groups.

To overcome these limitations, new ways to functionalize useful substrates with tetrazines have arisen in the last two decades, employing modern metal-catalyzed cross-coupling reactions (Figure 1). A potential approach is to use (aryl)tetrazine derivatives, in which the $\mathrm{C}-\mathrm{C}$ bond formation reaction takes place onto the aryl substituent. ${ }^{22-24}$ 
Alternatively, direct coupling at position 3 or 6 of the tetrazine ring is preferable because it leads to the introduction of minimal perturbation in the attached substrate. The first example on direct tetrazine cross-coupling employed the Sonogashira reaction between terminal alkynes and electronically enriched chloro-tetrazines. ${ }^{25}$ Unfortunately, the tertiary amine substituent in the reported tetrazines limited their bioorthogonal applications. Importantly, if the tetrazine ring has an electron-donating substituent, the subsequent bioorthogonal iEDDA cycloaddition tends to suffer from slow reaction kinetics, which is critical for their use in vivo. ${ }^{26}$ Suzuki and Stille-type couplings with chloro27,28 and methylthio-tetrazines ${ }^{29}$ have been reported, but only to generate 3-alkylamino-6-aryl-1,2,4,5-tetrazines, which suffer the same drawback in terms of slow cycloaddition kinetics (Figure 1).

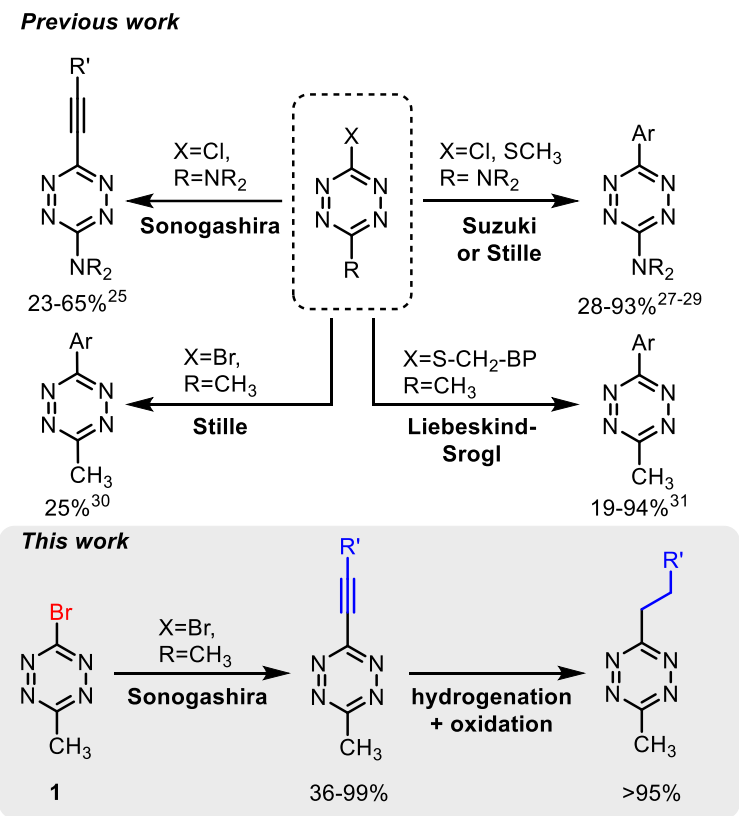

Figure 1: Overview of the reported metal-catalysed cross-couplings with 1,2,4,5-tetrazines.

An improvement in the bioorthogonal applicability for the products of these reactions was reported when a set of fluorescein and Oregon green derivatives were synthesized via a Stille coupling, employing 3-bromo-6-methyl1,2,4,5-tetrazine (1) as reagent. ${ }^{30}$ The obtained dyes were successfully utilized in no-wash live-cell imaging. However, the resulting (aryl)tetrazines were obtained in low yields, and no examples with alkyl substituents in the tetrazine ring were described. Also, the relatively inefficient synthesis reported for 1 ( $2 \%$ overall yield from the thiocarbohydrazide starting material) is a limitation for the broader use of this synthetic approach. Recently, the 6methyltetrazin-3-yl fragment has also been introduced via a Ag-mediated Liebeskind-Srogl reaction to arylboronic acids with excellent yields. ${ }^{31}$

When designing tetrazines for bioorthogonal purposes, which is the major application nowadays for these class of compounds, a compromise between reactivity and stability is needed. In general, the presence of electronwithdrawing groups (EWG) in the tetrazine ring increases the reactivity of tetrazines in the iEDDA cycloaddition by lowering their lowest unoccupied molecular orbital (LUMO). ${ }^{32}$ However, this is detrimental to their stability under physiological conditions, critically limiting their potential use in vivo. On the other hand, tetrazines with electrondonating groups (EDGs) are much more stable but react with sluggish kinetics, also constraining their use as bioorthogonal tools. Therefore, we envisaged that 3-alkyl-6-methyl-1,2,4,5-tetrazines might strike a balance between stability and reactivity, improving their potential for many applications in chemical biology. ${ }^{30,31,33-35}$ 
The revision of existing literature on cross-coupling reactions at position 3- or 6- of the tetrazine ring seems to confirm that chlorine is more inert than bromine as the halogen, ${ }^{36}$ thus requiring electronically enriched tetrazines and strong reaction conditions; in contrast, bromine can react under milder conditions, and a larger number of interesting substrates can potentially be accessed. ${ }^{30}$ Consequently, we directed our efforts towards the improvement of the overall synthetic route for 1.

Herein, we describe an optimized synthesis of 1 , achieving a $15 \%$ overall yield, the highest reported to date for this reagent. ${ }^{30,37}$ We also describe the use of 1 in the Sonogashira couplings with terminal alkynes and the synthesis of 3-alkyl-6-methyl-1,2,4,5-tetrazines via hydrogenation and re-oxidation of the alkynyl derivatives, reaching unprecedented yields for this type of compounds. The methodology has been applied to the synthesis of several unnatural amino acids bearing the 6-methyltetrazyn-3-yl fragment in their side chain.

\section{Results and discussion}

Although 1 was first described in $1978,{ }^{37}$ its use as a reagent to introduce the tetrazine fragment was hampered by the low yields of the previously reported syntheses. ${ }^{30,37}$ We followed a synthetic route analogous to the one recently published by us to generate unsubstituted 3-bromo-1,2-4,5-tetrazine. ${ }^{17}$ Thiocarbohydrazide was alkylated with 1 iodododecane to afford the iodide salt $\mathbf{2}$, which was then reacted with triethylorthoacetate in the presence of triethylamine to generate 3-(dodecylthio)-6-methyl-1,2,4,5-tetrazine (3) (Scheme 1). Notably, 3 was formed at room temperature and without the need for any oxidizing agent. Without further purification, intermediate $\mathbf{3}$ was reacted with a solution of hydrazine in THF to achieve the hydrazinyl-methyltetrazine 4, which was finally brominated to achieve the final product 1 as a red, crystalline solid. This synthesis, in addition to the larger overall yield (15\%) compared to the highest reported to date $(2 \%),{ }^{30}$ has the advantages of being scalable, with non-volatile intermediates, and not requiring any metal catalysis or oxidants.

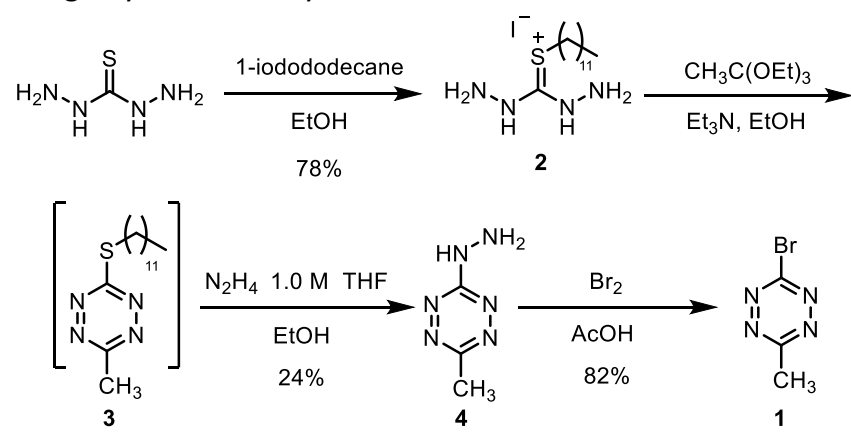

Scheme 1: Synthesis of 3-bromo-6-methyl-1,2,4,5-tetrazine 1.

With an efficient synthesis of $\mathbf{1}$ in hand, we then focused on screening different Pd-catalyzed reactions, such as Suzuki cross-coupling or Miyaura borylation. These initial attempts evidenced that 1 underwent undesired reactions when using inorganic bases, and decomposed at high reaction temperatures. Hence, we decided to test a Sonogashira-type reaction, as it normally requires neither of these two factors. Taking as a reference previous work on this reaction with halogenated tetrazines, ${ }^{25}$ we decided to test the coupling between 1 and trimethylsilylacetylene. It had been previously reported that all attempts to perform a Sonogashira reaction with this substrate and electronically enriched chlorotetrazines proved unsuccessful and only decomposition could be observed. ${ }^{25}$ We envisaged that if we could achieve the formation of the coupled product 3-methyl-6((trimethylsilyl)ethynyl)-1,2,4,5-tetrazine (5a), it would be a good starting point to explore further the stability and reactivity of other 3-ethynyl-6-methyl-1,2,4,5-tetrazines. Using $5 \mathrm{~mol} \%$ of $\mathrm{Pd}^{\prime \prime}$ precatalysts (either $\mathrm{PdCl}{ }_{2}$ or $\mathrm{Pd}(\mathrm{OAc})_{2}$ ) (table 1, entries 1 and 2) in toluene, the desired product 5 a was formed in moderate yields. The use of $\mathrm{Pd}(\mathrm{PPh})_{4}$ as 
catalyst resulted in lower yields (table 1, entry 3). However, an excellent $75 \%$ yield was obtained using $\mathrm{PdCl}_{2}\left(\mathrm{PPh}_{3}\right)_{2}$ (table 1, entry 4). Using more polar solvents, like dichloromethane or tetrahydrofurane (table 1, entries 5 and 6 ) or increasing the temperature (table 1, entries 7 and 8), worsened the yields. We concluded that, under mild conditions, bromotetrazine 1 is an excellent reagent to undergo Sonogashira couplings with terminal alkynes.

Table 1. Screening of reaction conditions for Sonogashira coupling of 1.

\begin{tabular}{|c|c|c|c|c|c|}
\hline & $+\equiv$ Si- & $2 \mathrm{eq}$ & $\begin{array}{l}\text { talyst } \\
1 \% \mathrm{PPh}_{3} \\
\text { ol \% Cul } \\
\text { opropylamin } \\
\text { vent, } \mathrm{T} \\
\text { ernight }\end{array}$ & $\mathrm{N}=\mathrm{N}$ & \\
\hline Entry & Catalyst & $\mathrm{T}$ & Solvent & Conv. [\%] ${ }^{[a]}$ & Yield [\%] $]^{[\mathrm{b}]}$ \\
\hline 1 & $\mathrm{PdCl}_{2}$ & r.t. & Toluene & $>99$ & 57 \\
\hline 2 & $\mathrm{Pd}(\mathrm{OAc})_{2}$ & r.t. & Toluene & $>99$ & 54 \\
\hline 3 & $\mathrm{Pd}\left(\mathrm{PPh}_{3}\right)_{4}$ & r.t. & Toluene & $>99$ & 23 \\
\hline 4 & $\mathrm{PdCl}_{2}\left(\mathrm{PPh}_{3}\right)_{2}$ & r.t. & Toluene & $>99$ & 75 \\
\hline 5 & $\mathrm{PdCl}_{2}\left(\mathrm{PPh}_{3}\right)_{2}$ & r.t. & DCM & $>99$ & 56 \\
\hline 6 & $\mathrm{PdCl}_{2}\left(\mathrm{PPh}_{3}\right)_{2}$ & r.t. & THF & $>99$ & 5 \\
\hline 7 & $\mathrm{PdCl}_{2}\left(\mathrm{PPh}_{3}\right)_{2}$ & 50 & Toluene & $>99$ & 66 \\
\hline 8 & $\mathrm{PdCl}_{2}\left(\mathrm{PPh}_{3}\right)_{2}$ & 100 & Toluene & $>99$ & 53 \\
\hline
\end{tabular}

The reaction was performed in a degassed, sealed vial with $0.057 \mathrm{mmol}$ of 1 [0.15 M], using $5 \mathrm{~mol} \%$ of catalyst loading and 2 eq. of TMS-acetylene as the model alkyne. [a] Determined by ${ }^{1} \mathrm{H}$ NMR spectroscopy. [b] Yield determined by $\mathrm{H}^{1} \mathrm{NMR}$ spectroscopy using mesitylene as an internal standard.

With the optimized reaction conditions in hand, we explored the substrate scope using a wide range of terminal alkynes (Scheme 2). Aliphatic alkynes afforded acetylenic tetrazines $\mathbf{5 b}$ - $\mathbf{d}$ in good to excellent yields. Likewise, aromatic alkynes with different substitution patterns (phenyl, tolyl, $p$-chlorophenyl, $o$ - and $p$-methoxyphenyl) were tested yielding the desired products (5e-i) in moderate to good yields. As it could be anticipated, the coupling with nucleophilic substrates (alcohols or amines) did not afford desired products, giving mainly the nucleophilic aromatic substitution products. Likewise, free carboxylic acids did not yield the desired products, leading to decomposition of the tetrazine ring. Therefore, we needed to use protecting groups. Acetylenic amines were protected either as phthalimides $(\mathbf{5} \mathbf{j})$ or butyl carbamates $\mathbf{5} \mathbf{k})$, affording the coupling products in good to moderate yields. Likewise, we successfully protected a hydroxyl as tetrahydropyranyl ether (5I). Finally, several acetylenic esters similar to $\mathbf{5 m}$ were tested with good results. 


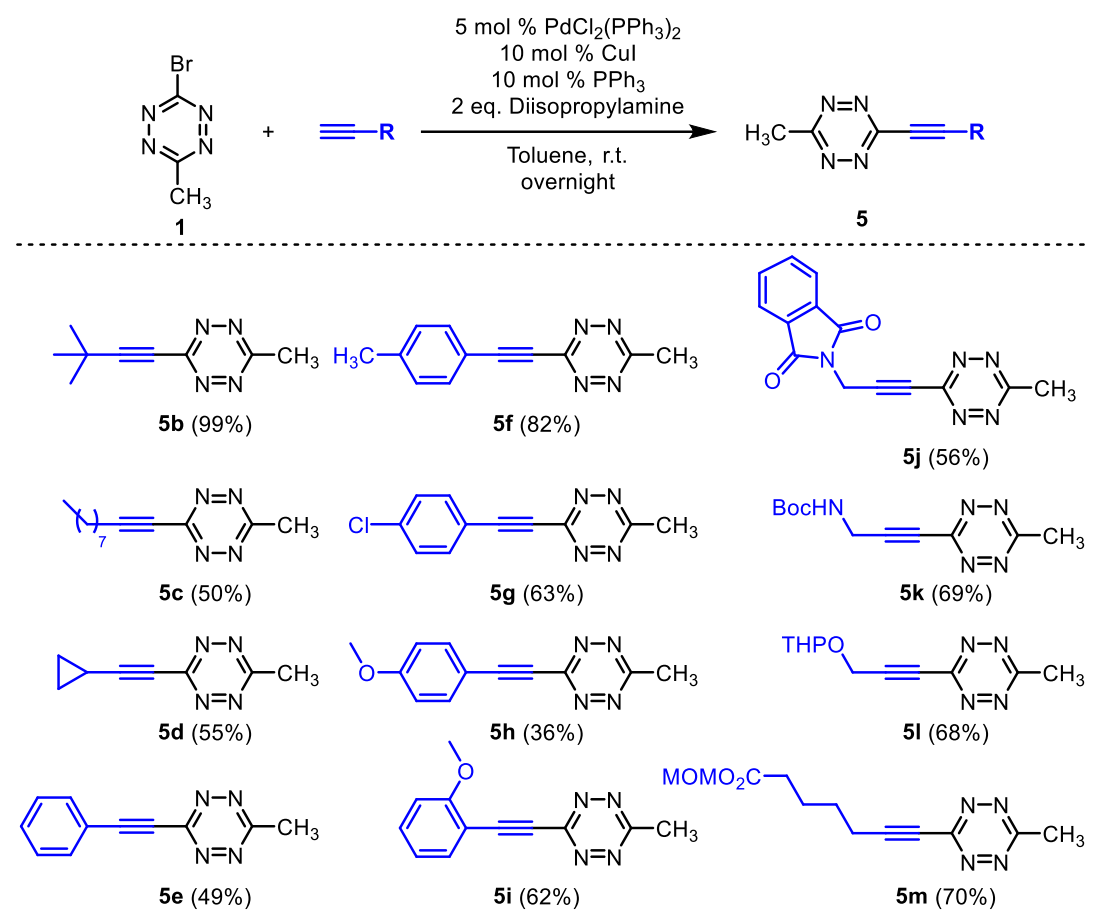

Scheme 2. Substrate scope of the Sonogashira reaction between 1 and aliphatic and aromatic alkynes.

The next step was to find good deprotection conditions for compounds $\mathbf{5 j}-\mathbf{m}$. We soon realized that basic conditions were not compatible with the tetrazine ring. Thus, phthalimide deprotection of compound $\mathbf{5} \mathbf{j}$ with hydrazine did not yield the desired product. Therefore, we studied a range of acidic conditions. Using a diluted solution of $\mathrm{HCl}$, deprotection of Boc-amine $\mathbf{5 k}$ was successful, although we were not able to isolate the corresponding free amine $\mathbf{6 a}$ (Scheme 3). The tetrahydropyranyl ether $\mathbf{5 I}$ was also successfully deprotected under similar mild acidic conditions to give the subsequent free alcohol $\mathbf{6 b}$. In the case of carboxylic acids, we studied the acidic ester hydrolysis. However, methyl and tert-butyl esters could only be removed through addition of water to the reaction conditions, which proved deleterious for the tetrazine ring and led to degradation. This problem could be circumvented by the use of the unconventional methoxymethyl (MOM) esters. Thus, compound $\mathbf{5 m}$ could be deprotected under the same mild conditions as for the protected alcohols and amines to obtain the free carboxylic acid $6 c$ (Scheme 3).

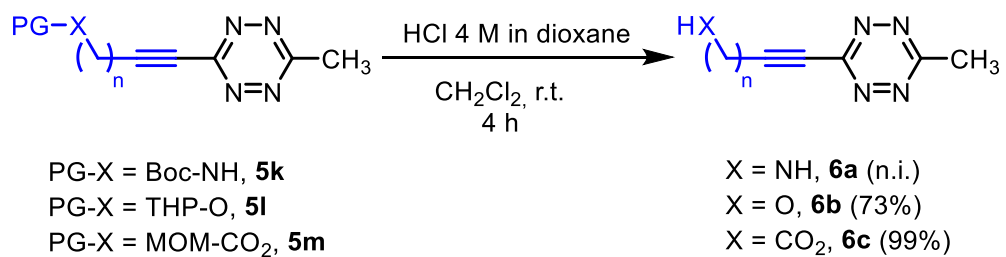

Scheme 3: Deprotection of acid-labile protective groups for amines, alcohols and carboxylic acids. n.i.: compound was not isolated.

Even though alkynyl-tetrazines 5a-m are an interesting new class of compounds which hold a potential use in bioorthogonal iEDDA reactions, we envisaged they could also be useful intermediates to generate asymmetrically substituted (dialkyl)tetrazines by hydrogenation of the triple bond. 3-alkyl-6-methyl-1,2,4,5-tetrazines are a synthetically challenging class of compounds and, to date, the only reliable approach for their synthesis is based on the condensation of two nitriles with anhydrous hydrazine, catalysed by $\mathrm{Ni}$ " or $\mathrm{Zn}$ " (in the form of salts, such as 
$\mathrm{Ni}(\mathrm{OTf})_{2}$ or $\left.\mathrm{Zn}(\mathrm{OTf})_{2}\right)$ (Figure 2). These metal ions act as Lewis acids, coordinating to the starting alkyl nitriles and activating them. ${ }^{21}$ The obtained dihydrotetrazines must be oxidized in situ to afford the resulting tetrazines in low or moderate yields. This method normally involves anhydrous hydrazine, the commercialisation of which is restricted in Europe or China due to safety concerns. ${ }^{38}$ Moreover, hydrazine can also act both as a good nucleophile and reductant, which can bring some functional group intolerabilities, specially under heating. ${ }^{38}$

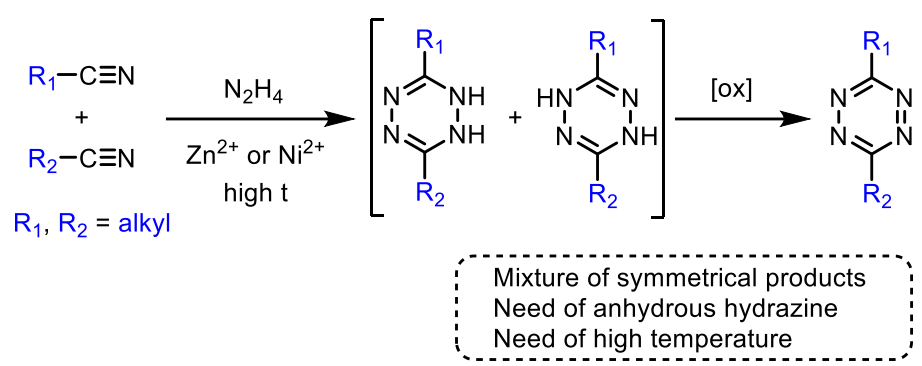

Figure 2: Synthetic approach for the synthesis of asymmetrically substituted (alkyl)tetrazines.

Due to these limitations, we decided to test the hydrogenation of the triple bond on two of the Sonogashira coupling products (5c and $\mathbf{5 f}$, Scheme $\mathbf{4}$ ), which were used as model compounds. When each of them was subjected to hydrogenation at 1 bar $(\mathrm{g})$ of pressure, using $\mathrm{Pd} / \mathrm{C}$ as catalyst, the characteristic pink coloration of tetrazines disappeared almost immediately in both cases. ${ }^{1} \mathrm{H}$ NMR spectroscopy of the reaction crude showed a mixture of compounds present, which can be consistent with the formation of the different dihydrotetrazine isomers. When we attempted the re-oxidation to the fully functional tetrazine on the reaction crude employing the typical $\mathrm{NaNO}_{2} / \mathrm{H}^{+}$ preparation -the most usual way to oxidize dihydrotetrazines ${ }^{26}$ we observed the formation of the desired product with moderate yields, together with degradation products. Gratifyingly, when the re-oxidation was performed with an hypervalent iodine reagent [(diacetoxyiodo)benzene], ${ }^{30}$ full conversion to the desired products $\mathbf{7 a}$ and $\mathbf{7 b}$ was achieved. The two-step sequence of hydrogenation/oxidation gave almost quantitative yields.
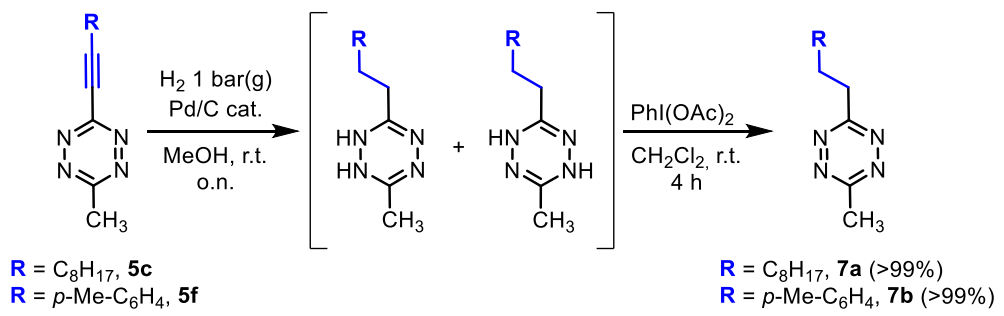

Scheme 4: Stepwise hydrogenation/re-oxidation in alkynyltetrazines $\mathbf{5 c}$ and $\mathbf{5 f}$ taken as model (aliphatic and aromatic) compounds.

Finally, to showcase the versatility of the reported synthetic strategy on biologically relevant molecules, the synthesis of different unnatural amino acids was performed (Scheme 5). Tetrazine-containing amino acids are interesting compounds in chemical biology, and several have been genetically incorporated in proteins in vivo. $17,33,34,39,40$ Therefore, following our previous findings, N-Boc and MOM esters were selected as appropriate protecting groups. Thus, N-Boc protected MOM esters of L-propargylglycine (8a) and 4-ethynyl-L-phenylalanine (8b) were synthesised and reacted with 1 under the optimized Sonogashira conditions to afford the methyltetrazinylamino acid derivatives 9a-b. Interestingly, heating the reaction mixture at 50ㄷ was necessary in both cases, as only starting material was recovered when performing this reaction at room temperature. The direct deprotection of these intermediates under acidic conditions yielded the hydrochloride salts $\mathbf{1 0} \mathbf{a}-\mathbf{b}$, containing an alkynyl-tetrazine functionality. On the other hand, when intermediates $\mathbf{1 0 a - b}$ were subjected to the sequence of hydrogenation and 
re-oxidation, followed by deprotection of the Boc carbamate and MOM ester, the novel (dialkyl)tetrazine amino acids 12a-b were obtained.

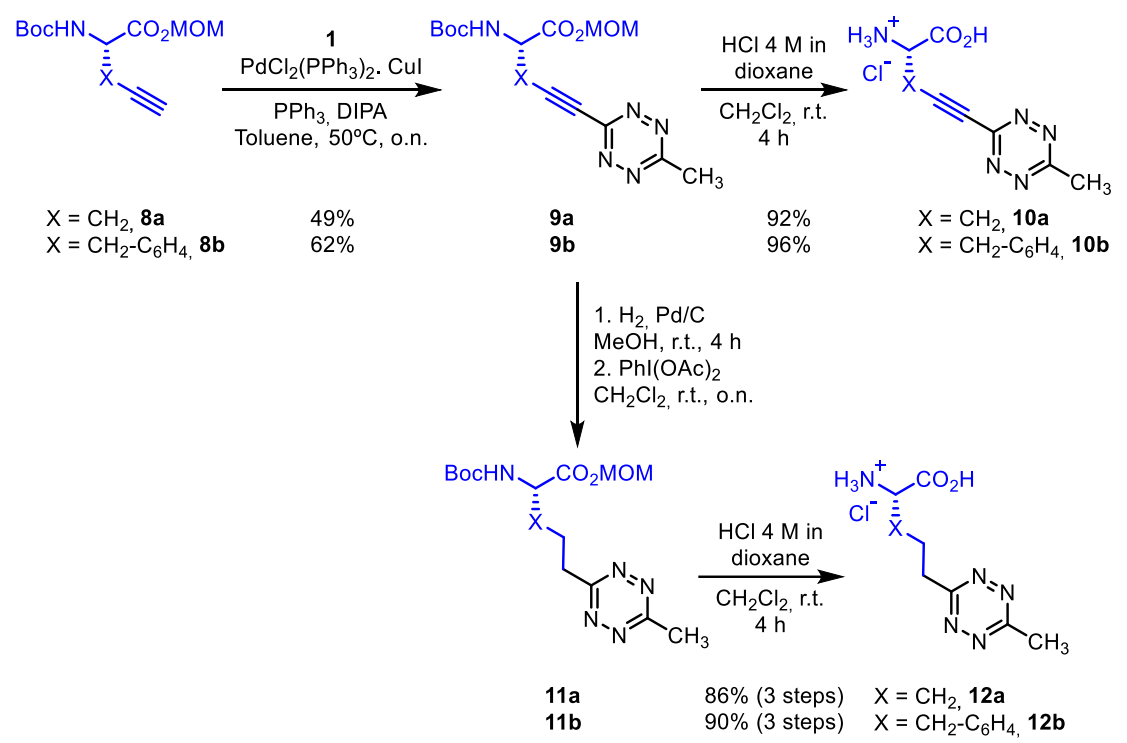

Scheme 5. Synthesis of unnatural amino acids bearing the 6-methyltetrazin-3-yl fragment.

\section{Conclusions}

In summary, we report an optimised synthetic route to 3-bromo-6-methyl-1,2,4,5-tetrazine 1, a useful tetrazine precursor to undergo Sonogashira-type cross-couplings with a set of structurally different terminal alkynes. The obtained products can be subjected to a sequence of alkyne hydrogenation and re-oxidation of the dihydrotetrazine ring to afford asymmetrically substituted (dialkyl)tetrazines in excellent yields. The versatility of the different reactions, together with the functional group tolerance provided by easily removable protective groups, enables the synthesis of a set of structurally different tetrazine-containing unnatural amino acids.

We anticipate this methodology can be further exploited to afford numerous new 1,2,4,5-tetrazines derived from the large catalogue of available alkynes, thereby achieving a wide range of potentially interesting compounds for chemical biology applications.

\section{Conflicts of interest}

There are no conflicts to declare.

\section{Acknowledgements}

This work was supported by grants from FEDER/Ministerio de Ciencia, Innovación y Universidades (MICINN)Agencia Estatal de Investigación (CTQ2017-87840-P to A.R.) and the Spanish Ministry of Economy, Industry and Competitiveness (MINECO, BIO2015-64572-R to L.R.dP). IRB Barcelona is the recipient of institutional funding from MICINN through the Centres of Excellence Severo Ochoa award and from the CERCA Program of the Catalan Government E.R. thanks the support of funding from La Caixa Foundation (ID 100010434) and La Caixa Ph.D. Fellowship. 


\section{Notes and references}

S. C. Benson, L. Lee, L. Yang and J. K. Snyder, Tetrahedron, 2000, 56, 1165-1180.

D. L. Boger and J. Hong, J. Am. Chem. Soc., 2001, 123, 8515-8519.

W. Kaim, Coord. Chem. Rev., 2002, 230, 127-139.

D. E. Chavez and M. A. Hiskey, J. Energ. Mater., 1999, 17, 357-377.

D. E. Chavez and M. A. Hiskey, J. Heterocycl. Chem., 1998, 35, 1329-1332.

H. C. Kolb, M. G. Finn and K. B. Sharpless, Angew. Chem., Int. Ed., 2001, 40, 2004-2021.

H. C. Kolb and K. B. Sharpless, Drug Discov. Today, 2003, 8, 1128-1137.

M. L. Blackman, M. Royzen and J. M. Fox, J. Am. Chem. Soc., 2008, 130, 13518-13519.

N. K. Devaraj, R. Weissleder and S. A. Hilderbrand, Bioconjug. Chem., 2008, 19, 2297-2299.

G. B. Cserép, A. Herner and P. Kele, Methods Appl. Fluoresc., 2015, 3, 042001.

J. Šečkutè, J. Yang and N. K. Devaraj, Nucleic Acids Res., 2013, 41, e148-e148.

N. K. Devaraj, G. M. Thurber, E. J. Keliher, B. Marinelli and R. Weissleder, Proc. Natl. Acad. Sci., 2012, 109, 4762-4767.

A. Rutkowska, D. W. Thomson, J. Vappiani, T. Werner, K. M. Mueller, L. Dittus, J. Krause, M. Muelbaier, G. Bergamini and M. Bantscheff, ACS Chem. Biol., 2016, 11, 2541-2550.

T. Reiner and B. M. Zeglis, J. Label. Compd. Radiopharm., 2014, 57, 285-290.

H. Lebraud, D. J. Wright, C. N. Johnson and T. D. Heightman, ACS Cent. Sci., 2016, 2, 927-934.

X. Ji, Z. Pan, B. Yu, L. K. De La Cruz, Y. Zheng, B. Ke and B. Wang, Chem. Soc. Rev., 2019, 48, $1077-1094$.

E. Ros, M. Bellido, X. Verdaguer, L. Ribas de Pouplana and A. Riera, Bioconjug. Chem., 2020, 31, 933-938.

B. L. Oliveira, Z. Guo and G. J. L. Bernardes, Chem. Soc. Rev., 2017, 46, 4895-4950.

S. Mayer and K. Lang, Synthesis, 2016, 49, 830-848.

G. Clavier and P. Audebert, Chem. Rev., 2010, 110, 3299-3314.

J. Yang, M. R. Karver, W. Li, S. Sahu and N. K. Devaraj, Angew. Chem., Int. Ed., 2012, 51, 5222-5225.

H. Xiong, Y. Gu, S. Zhang, F. Lu, Q. Ji, L. Liu, P. Ma, G. Yang, W. Hou and H. Xu, Chem. Commun., 2020, 56, 4692-4695.

A. Wieczorek, T. Buckup and R. Wombacher, Org. Biomol. Chem., 2014, 12, 4177-4185.

G. Knorr, E. Kozma, A. Herner, E. A. Lemke and P. Kele, Chem. Eur. J., 2016, 22, 8972-8979.

Z. Novák and A. Kotschy, Org. Lett., 2003, 5, 3495-3497.

B. L. Oliveira, Z. Guo and G. J. L. Bernardes, Chem. Soc. Rev., 2017, 46, 4895-4950.

F. Pop, J. Ding, L. M. L. Daku, A. Hauser and N. Avarvari, RSC Adv., 2013, 3, 3218-3221.

A. M. Bender, T. C. Chopko, T. M. Bridges and C. W. Lindsley, Org. Lett., 2017, 19, 5693-5696.

N. Leconte, A. Keromnes-Wuillaume, F. Suzenet and G. Guillaumet, Synlett, 2007, 204-210.

A. Wieczorek, P. Werther, J. Euchner and R. Wombacher, Chem. Sci., 2017, 8, 1506-1510.

W. D. Lambert, Y. Fang, S. Mahapatra, Z. Huang, C. W. Am Ende and J. M. Fox, J. Am. Chem. Soc., 2019, 141, 17068-17074.

R. A. A. Foster and M. C. Willis, Chem. Soc. Rev., 2013, 42, 63-76.

R. J. Blizzard, D. R. Backus, W. Brown, C. G. Bazewicz, Y. Li and R. A. Mehl, J. Am. Chem. Soc., 2015, 137, 10044-10047.

S. V. Mayer, A. Murnauer, M. K. von Wrisberg, M. L. Jokisch and K. Lang, Angew. Chem., Int. Ed., 2019, 58, 15876-15882.

M. R. Karver, R. Weissleder and S. A. Hilderbrand, Bioconjug. Chem., 2011, 22, 2263-2270. 
39 J. L. Seitchik, J. C. Peeler, M. T. Taylor, M. L. Blackman, T. W. Rhoads, R. B. Cooley, C. Refakis, J. M. Fox and R. A. Mehl, J. Am. Chem. Soc., 2012, 134, 2898-2901. 